\title{
Formation Flight Control
}

\author{
Yu Gu, ${ }^{1}$ Giampiero Campa, ${ }^{2}$ and Mario Innocenti ${ }^{3}$ \\ ${ }^{1}$ Department of Mechanical and Aerospace Engineering, West Virginia University, Morgantown, WV 26506, USA \\ ${ }^{2}$ The Mathworks, El Segundo, CA 90245, USA \\ ${ }^{3}$ Department of Electrical Systems and Automation, University of Pisa, 56126 Pisa, Italy
}

Correspondence should be addressed to Yu Gu, yu.gu@mail.wvu.edu

Received 27 June 2011; Accepted 27 June 2011

Copyright (C) $2011 \mathrm{Yu} \mathrm{Gu}$ et al. This is an open access article distributed under the Creative Commons Attribution License, which permits unrestricted use, distribution, and reproduction in any medium, provided the original work is properly cited.

The formation flight control problem has been extensively discussed in recent years with numerous applications on aircraft and spacecraft systems. For aerial vehicles, advantages of performing formation flight have been well documented, including fuel saving, improved efficiency in air traffic control, and cooperative task allocation. For space vehicles, precise control of formation flight will enable future large-aperture space telescopes, variable baseline space interferometers, autonomous rendezvous and docking, and robotic assembly of space structures.

To facilitate further development in this emerging area of research, this special issue includes several papers discussing the most recent developments and ideas in the field, with emphasis on both the theoretical and experimental results. Of the five papers selected, three are in the area of spacecraft formation flight control and two are related to autonomous aerial vehicles. Contents of these papers include modeling and estimation, guidance, navigation, and control (GNC), and experimental techniques related to autonomous formation flight.

The first paper of this special issue "Guidance navigation and control for autonomous multiple spacecraft assembly: analysis and experimentation" explores the GNC problem for the in-plane orbital assembly of autonomous multiple spacecraft. The guidance and control strategies are designed to take into account the evolving shape and mass properties of the assembling spacecraft. The proposed approaches were validated via hardware-in-the-loop experiments, using four autonomous 3-degree-of-freedom robotic spacecraft simulators.

The second paper "Cascades-based controlled attitude synchronization and tracking of spacecraft in leader-follower formation" presents control strategies for leader-follower attitude synchronization of spacecraft formations in the presence of disturbances. In a leader-follower formation configuration, the leader spacecraft is controlled to follow a given reference, while a follower spacecraft is controlled to synchronize its motion with the leader. A stability analysis is provided in the paper for both known and unknown but bounded disturbances.

The third paper "Design of an extended interacting multiple models adaptive estimator (EIMMAE) for attitude determination of a stereo-imagery satellite" deals with the attitude determination issue for a pair of satellites used in a stereoimaging scenario. For this type of operations, highly accurate and stable pointing maneuvers are needed to be accomplished in a few seconds, which requires the satellites to rotate along a relatively large-angle attitude very quickly. Therefore, different estimation strategies are discussed for two different modes: "maneuvering motion" mode and "uniform motion" mode.

The fourth paper "Decentralized model predictive control for cooperative multiple vehicles subject to communication loss" investigates the control of multiple cooperative vehicles with the possibility of communication loss/delay. Such communication issues could lead to poor cooperation performance and unsafe behaviors such as collisions. A decentralized model predictive control (DMPC) architecture is proposed to estimate the tail of neighbor's trajectory which is not available due to the large communication delays. The concept of the tube MPC is also employed to improve the safety of the fleet against collisions, in the presence of large intervehicle communication delays.

The final paper of this special issue "Vision-based tracking of uncooperative targets" presents both the flight test and simulation results of a follower aircraft tracking an 
uncooperative leader, using only monocular vision information. The situations with and without the subtended angle information for range estimation are both discussed with different approaches presented.

$Y u G u$ Giampiero Campa Mario Innocenti 

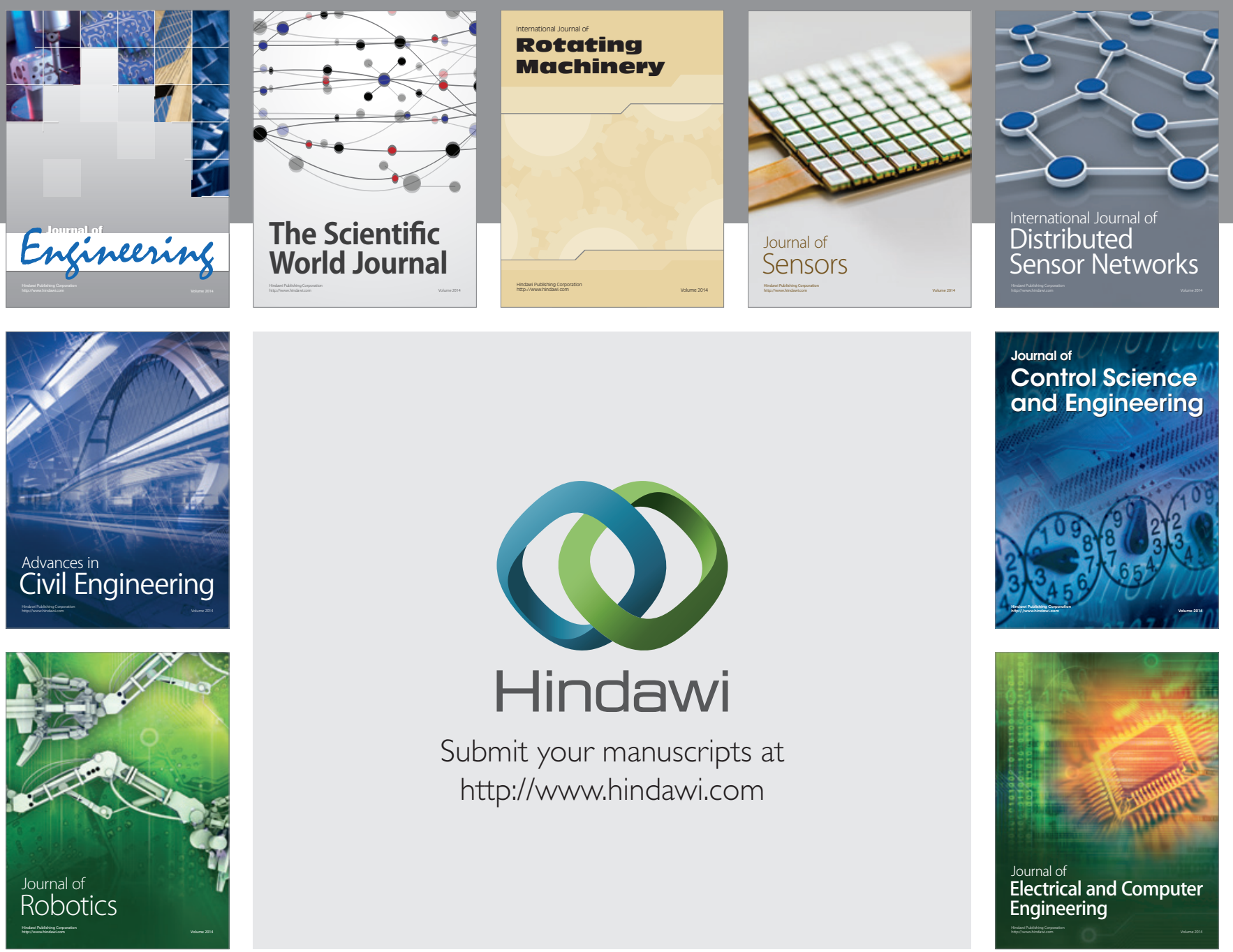

Submit your manuscripts at

http://www.hindawi.com
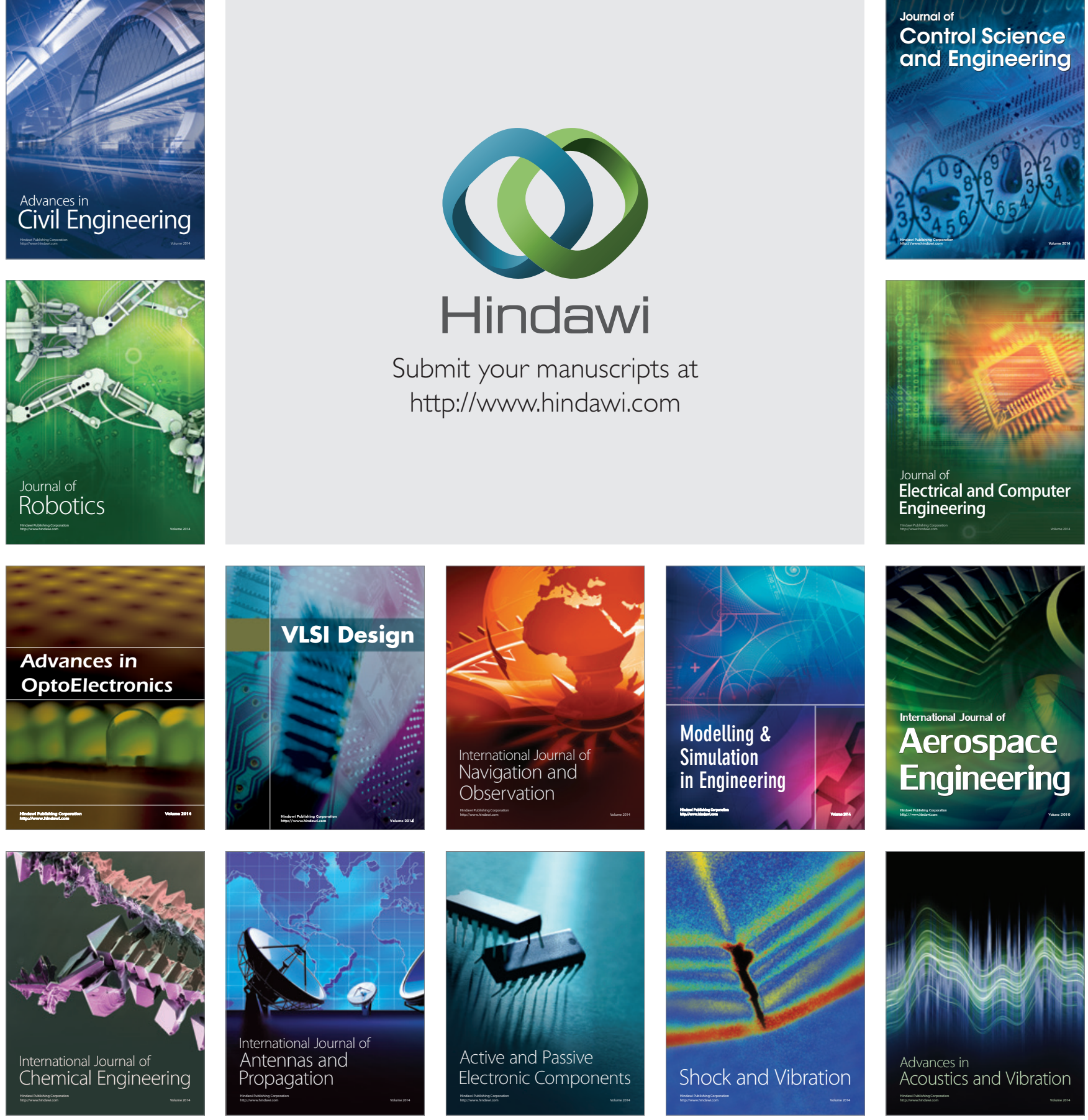